\title{
Évolution des déterminants des maladies chroniques du foie au Québec
}

\author{
A. J. Sanabria, M. Sc. (1, 2); R. Dion, M.D. (3); E. Lúcar, B. Sc. (1, 4); J. C. Soto, M.D. (1)
}

Cet article a fait l'objet d'une évaluation par les pairs.

\section{Résumé}

Introduction : Les maladies chroniques du foie (MCF) constituent un problème mondial. La morbidité et la mortalité des MCF pourraient être évitées ou atténuées en agissant sur leurs principaux facteurs de risque, notamment l'obésité et la consommation d'alcool.

Méthodologie : Afin de décrire l'évolution des principaux déterminants des MCF au Québec, nous avons étudié les tendances de l'obésité, de la consommation d'alcool, des hépatites virales $\mathrm{B}$ et $\mathrm{C}$, de la mortalité et du taux d'hospitalisation des MCF ainsi que du taux d'incidence du cancer du foie entre le $1^{\text {er }}$ janvier 1981 et le 31 décembre 2009.

Résultats : Nous avons observé une augmentation des indicateurs de l'obésité chez les jeunes hommes et de la consommation d'alcool chez les adolescentes et chez les femmes d’âge moyen. Les taux d'incidence globale des hépatites B et C et de la mortalité par MCF sont en diminution. Cependant, les taux d'incidence du cancer du foie et de sa mortalité, surtout chez les hommes et les personnes âgées, sont à la hausse.

Conclusion : Ces résultats soulignent l'importance des interventions de santé publique ciblées ainsi que le maintien ou l'amélioration de l'accès aux soins pour les MCF.

Mots-clés : maladie hépatique, épidémiologie, maladie chronique, facteurs de risque, maladie chronique du foie

\section{Introduction}

Les maladies chroniques du foie (MCF) forment un groupe d'entités ayant différentes caractéristiques en termes de facteurs de risque, d'incubation, de latence, d'induction et d'état final du processus morbide. Les plus fréquentes sont celles associées aux hépatites virales chroniques, à la consommation d'alcool et à l'obésité; les moins communes sont le cancer du foie et celles dues à certaines conditions génétiques, auto-immunes, vasculaires ou à une toxicité pharmacologique ${ }^{1}$.

Les MCF sont responsables de plus de $80 \%$ des cancers primitifs du foie, dont le carcinome hépatocellulaire $(\mathrm{CHC})$ représente la forme la plus fréquente. L'inflammation chronique du foie et la fibrose du tissu hépatique fournissent les conditions idéales à son développement. Ainsi, les patients atteints de $\mathrm{CHC}$ présentent habituellement des antécédents d'hépatites virales B (VHB) ou C (VHC) chroniques, de maladie alcoolique du foie ou de stéatohépatite non alcoolique ${ }^{2,3}$. Seules ou en combinaison, ces maladies augmentent le risque de $\mathrm{CHC}^{4-8}$.

Les MCF constituent un problème de santé publique important au niveau mondial. En Angleterre, elles occupent la cinquième place parmi les causes de décès ${ }^{9}$, et aux
États-Unis, elles figurent parmi les quinze premières causes de décès dans la dernière décennie $\mathrm{e}^{10-13}$, avec un taux de mortalité ajusté estimé de 10,3 par 100000 habi$\operatorname{tants}^{13}$ en 2010. Au Canada, en 2008, 2748 décès ont été imputés aux MCF et à la cirrhose du foie $\left(11^{\mathrm{e}}\right.$ rang des principales causes de décès), dont 1809 (66 \%) chez les hommes ${ }^{14}$.

Un élément intéressant sur le plan de la santé publique est que la morbidité et la mortalité des MCF pourraient être évitées ou atténuées en agissant sur leurs principaux facteurs de risque, notamment l'obésité et la consommation d'alcool ${ }^{6-8}$. La vaccination contre le virus de l'hépatite $A$ et le VHB, le diagnostic et le traitement précoces de l'hépatite chronique causée par le VHC peuvent aussi permettre d'éviter certaines complications ${ }^{7,15,16}$.

Dans le but de connaître l'état de la situation des MCF au Québec, nous avons étudié leur évolution ainsi que celle de leurs principaux déterminants. Nous avons ciblé les affections pour lesquelles la prévention joue un rôle important.

\section{Méthodologie}

Notre étude d'observation de type écologique a été réalisée pour la période comprise entre le $1^{\text {er }}$ janvier 1981 et le 31 décembre 2009. Les indicateurs de santé choisis provenaient de l'Infocentre de santé publique (Infocentre) de l'Institut national de santé publique du Québec (INSPQ), à l'exception de ceux sur la morbidité associée aux VHB et VHC, lesquels provenaient du registre des mala-

Rattachement des auteurs :

1. Direction des risques biologiques et de la santé au travail, Institut national de santé publique du Québec (INSPQ), Montréal (Québec), Canada

2. Hôpital Universitaire de Bellvitge, Barcelone, Espagne

3. Laboratoire de santé publique du Québec, INSPQ, Sainte-Anne-de-Bellevue (Québec), Canada

4. Université McGill, Montréal (Québec), Canada

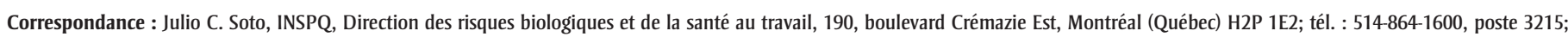
téléc. : 514-864-7646; courriel : julio.soto@inspq.qc.ca 
dies à déclaration obligatoire (MADO) du Québec. L'Infocentre est un portail d'accès sécurisé permettant l'exploitation de données sanitaires provenant de diverses sources $^{17}$.

Les indicateurs des déterminants des MCF choisis provenaient des Enquêtes sur la santé dans les collectivités canadiennes (ESCC) 2000-2001, 2003-2005 et 20072008, réalisées par Statistique Canada ${ }^{18}$. Ces enquêtes visent les personnes âgées de 12 ans et plus des ménages privés (excluant les résidents des réserves et d'autres peuplements autochtones, des bases des Forces armées canadiennes, des établissements et de certaines régions éloignées) et sont représentatives des régions sociosanitaires du Québec ${ }^{18}$.

Les données du registre MADO sur les cas déclarés de VHB et VHC couvraient la période du $1^{\mathrm{er}}$ janvier 1990 au 31 décembre 2009. Pour le VHB, nous avons retenu les nouvelles infections (cas aigus), les cas de porteur chronique et ceux dont le statut n'était pas précisé. Pour le VHC, toutes les classes confondues ont été retenues. Ces cas sont enregistrés et classés par les Directions de santé publique régionales du Québec selon les définitions nosologiques du ministère de la Santé et des Services sociaux $(\mathrm{MSSS})^{19}$, semblables à celles de l'Agence de la santé publique du Canada $(\mathrm{ASPC})^{20}$. Les taux d'incidence annuelle par 100000 habitants ont été calculés pour les infections au VHB aiguës en utilisant comme dénominateurs les estimés et les projections de l'Institut de la statistique du Québec (ISQ) effectués à partir des données des recensements de Statistique Canada et publiés par le $\mathrm{MSSS}^{21}$. Les données sur le VHB et le VHC ont été analysées au moyen du logiciel EpiData Analysis version 2.2.1.171 22 .

Les indicateurs étudiés et associés aux déterminants des MCF étaient les suivants : proportion de la population présentant un indice de masse corporelle (IMC) entre 25 et 29,9 (embonpoint) et proportion de la population présentant un IMC supérieur ou égal à 30 (obésité) $^{23}$ pour la période 20002008; consommation d'alcool (proportion de buveurs au cours des douze derniers mois et proportion de personnes avec une fréquence de consommation quotidienne d'alcool) pour la période 2000-2008; proportion de la population ayant pris 14 consommations d'alcool et plus au cours d'une période de sept jours pour la période 2000-2005, et proportion de la population mentionnant une consommation élevée d'alcool (cinq verres d'alcool en une même occasion, soit un à la suite de l'autre ou dans une courte période de temps) 12 fois et plus au cours d'une année ${ }^{18}$ pour la période 2000-2008.

Les sources de données des indicateurs des MCF étaient le fichier des tumeurs et le fichier des décès du Québec du MSSS et le système de maintenance et d'exploitation de données pour l'étude de la clientèle hospitalière (MED-ÉCHO). Les codes des $9^{\mathrm{e}}$ et $10^{\mathrm{e}}$ versions de la classification internationale des maladies (CIM-9 et CIM-10) ont été utilisés pour identifier les MCF. La CIM-9 a été appliquée jusqu'à l'année 1999 (code 571 pour les MCF et code 115 pour le cancer du foie). À partir de l'année 2000, la CIM-10 a été utilisée (codes K70 pour la maladie alcoolique du foie et code K74 pour la cirrhose du foie).

Pour les MCF, les indicateurs utilisés étaient les suivants : taux de mortalité due aux MCF pour la période 1981-1999; taux de mortalité due à la cirrhose hépatique et taux de mortalité de la maladie alcoolique du foie pour la période 2000-2007; taux d'incidence pour le cancer du foie pour la période 1986-2007; taux de mortalité pour le cancer du foie pour la période 1981-2009 et taux d'hospitalisation en soins physiques de courte durée pour maladies du foie pour la période 1990-2005.

Les proportions des divers paramètres étudiés ainsi que les taux d'incidence, de mortalité et d'hospitalisation en établissements de soins de courte durée pour MCF ont été ajustés selon l'âge et le sexe à partir de la population de l'ensemble du Québec en 2001 et 2006. Les intervalles de confiance (IC) des estimés ont été calculés à $99 \%$.

\section{Résultats}

Au Québec, la proportion de la population âgée de 20 à 64 ans présentant un embonpoint a montré une légère tendance à la hausse, passant de 44,7\% (IC à $99 \%$ : 43,2 à 46,2) en 2000-2001 à 47,9\% (IC à $99 \%$ : 46,3 à 49,3) en 2007-2008. Pour les mêmes périodes, les chiffres globaux d'obésité pour le même groupe étaient aussi en augmentation, passant de 12,6\% (IC à $99 \%: 11,6$ à 13,7) à 15,8\% (IC à $99 \%$ : 14,6 à 17,0). Plus particulièrement, les hommes de 25 à 44 ans ont présenté une augmentation prononcée de leur taux d'obésité, qui est passé de 12,7\% (IC à $99 \%$ : 10,6 à 14,7) à 16,8 \% (IC à $99 \%$ : 14,1 à 19,6) (figure 1).

La proportion de buveurs (âgés de 12 ans et plus) a légèrement augmenté, passant de $81,1 \%$ en $2000-2001$ à $81,9 \%$ en 2007 2008. Cependant, lorsque l'on regarde la fréquence de consommation d'alcool quotidienne, la proportion de buveurs âgés de 12 ans est passé de 8,3\% (IC à $99 \%: 7,5$ à 9,1) en 2000-2001 à 10,7\% (IC à $99 \%$ : 9,8 à 11,5) en 2007-2008. Cette augmentation était plus évidente chez les femmes, passant de 4,6\% (IC à $99 \%: 4,1$ à 5,2) en 2000-2001 à 7,3 (IC $99 \%$ 6,3 à 8,3) en 2007-2008. Ces tendances à la hausse sont corroborées en observant les proportions de 14 consommations et plus d'alcool au cours d'une semaine par groupe d'âge et par sexe pour la période 2000-2005. Sauf exception, la hausse a été observée dans pratiquement tous les groupes d'âge étudiés, en particulier dans celui des 2024 ans chez les hommes. Chez les femmes, même si ces proportions étaient plus faibles que celles observées chez les hommes, les hausses les plus remarquables ont été observées dans les groupes des 45-64 ans, des 20-24 ans et des 1219 ans (figure 2).

Pour la consommation élevée d'alcool, la tendance à la hausse a été observée aussi bien chez les hommes que chez les femmes et pour presque toutes les tranches d'âge, à l'exception du groupe des 12-19 ans. Toutefois, c'est chez les femmes jeunes adultes (20-24 ans) que la hausse a été la plus marquée. Chez les hommes de cette tranche d'âge, la proportion est passée de 41,2 (IC à $99 \%: 33,7$ à 48,7) en 2000-2001 à 46,4 (IC à $99 \%: 37,8$ à 55,1) en 20072008 et chez les femmes de 17,1 (IC à $99 \%$ : 11,9 à 22,3) à 30,1 (IC à $99 \%: 22,9$ à 37,4) pour les mêmes cycles. 
FIGURE 1

Proportion de la population présentant une obésité selon l’âge et le sexe, à l'exclusion des femmes enceintes, Québec, ESCC, $2000-2008$

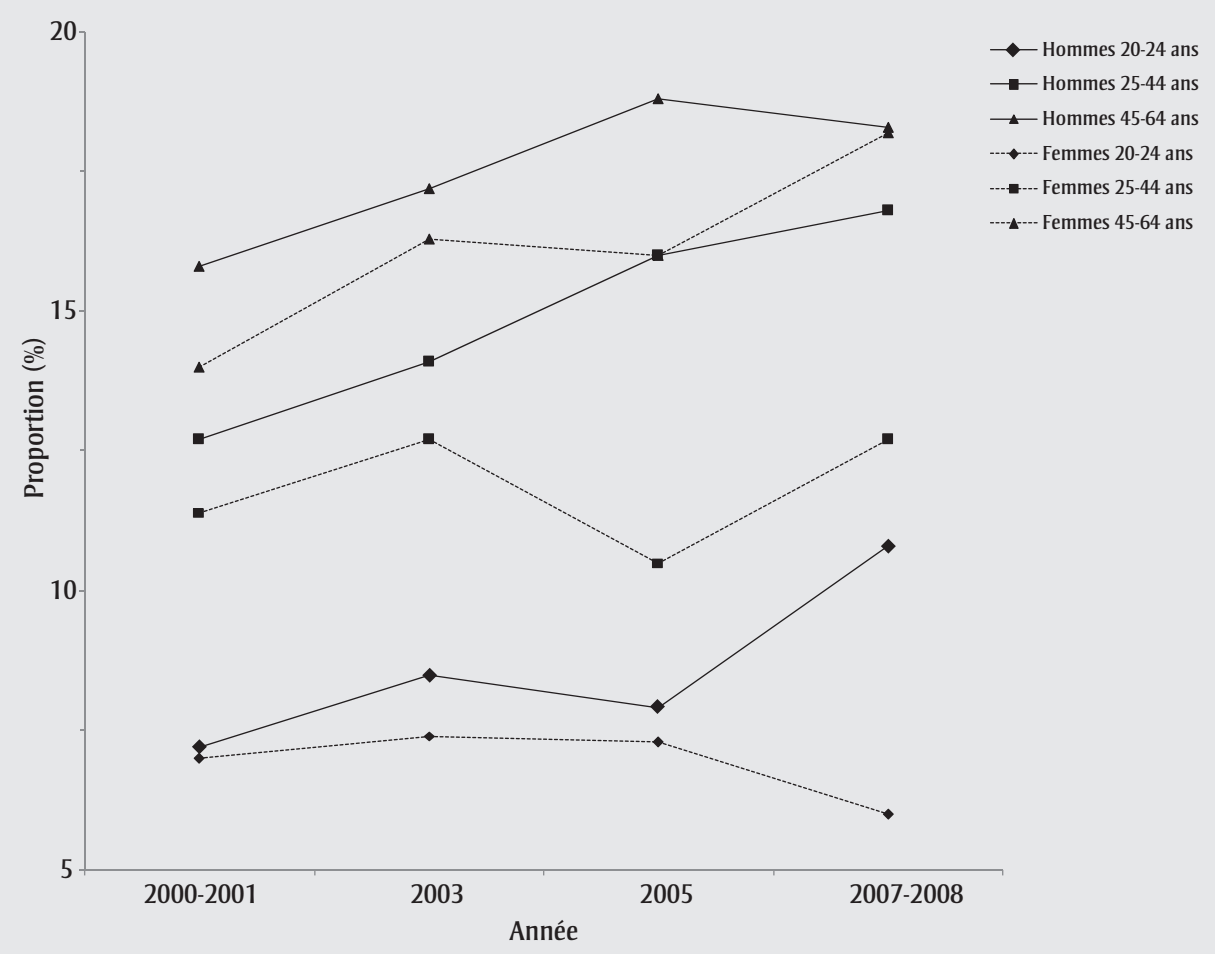

Abréviation : ESCC, Enquête sur la santé dans les collectivités canadiennes.

De 1990 à 2009, 28476 cas de VHB et 33490 cas de VHC ont été inscrits au registre MADO du Québec (figure 3). Parmi les infections au VHB, 14 \% ont été classées comme aiguës (nouvelles infections) et $71 \%$ comme affectant des porteurs chroniques; le statut n'a pas été précisé pour $15 \%$ des cas (cette proportion a augmenté depuis 2003). Le taux d'incidence globale des cas aigus a diminué de façon marquée depuis 1992, passant de 6,5 par 100000 à 0,4 par 100000 en 2009; cette réduction est survenue parallèlement chez les deux sexes et dans la plupart des groupes d'âge, mais particulièrement dans les groupes des 1019 ans et 20-39 ans (non illustré ici). Le nombre de porteurs chroniques déclarés a également diminué au cours de la même période, passant d'un maximum de 1434 cas en 1992 à 634 en 2009. Le nombre de cas d'infection par VHC a augmenté de façon marquée au cours des années 1990, pour diminuer graduellement par la suite.

Une diminution globale des taux de mortalité par MCF et par cirrhose du foie a été observée pour la période 1981-1999. Chez les hommes, le taux de mortalité due à ces affections est passé de 24,2 par 100000 (IC à $99 \%: 21,5$ à 27,3) en 1981 à 13,0 (IC à $99 \%$ : 11,3 à 14,9) en 1999, et chez les femmes de 8,2 par 100000 (IC à $99 \%: 6,8$ à 9,9) à 5,8 (IC à $99 \%: 4,8$ à 6,9) pour la même période (figure 4). Par ailleurs, le taux global de mortalité par cirrhose du foie est demeuré pratiquement stable pour la période 2000-2007; il est passé de 4,7 par 100000 en 2000 (IC à $99 \%: 4,0$ à 5,4 ) à 4,6 (IC à $99 \%: 4,0$ à 5,2) en 2007. Similairement, le taux de mortalité par maladie alcoolique du foie est passé de 3,3 par 100000 (IC à $99 \%$ : 2,8 à 3,9) à 3,1 (IC à $99 \%: 2,6$ à 3,6) pour les mêmes années (figure 5).

Par contre, une augmentation globale du taux d'incidence du cancer de foie a été observée entre 1986 et 2006 . Ce taux est passé de 3,8 par 100000 (IC à $99 \%$ : 3,4 à 4,2) pour 1986-1988 à 6,7 (IC à $99 \%: 6,7$ à 7,1) pour 2004-2006. Cette augmentation a touché les deux sexes : chez les femmes, ce taux est passé de 2,3 (IC à $99 \%: 1,9$ à 2,7) à 3,8 (IC à $99 \%: 3,4$ à 4,3) et chez les hommes, de 5,7 (IC à $99 \%: 5,0$ à 6,8) à 10,2 (IC à $99 \quad \% \quad: \quad 9,4$ à 11,0 ).
L'augmentation était remarquable à partir du groupe des 50-59 ans et a été plus importante chez les 80 ans et plus; pour eux, le taux est passé de 19,6 par 100000 (IC à $99 \%: 14,5$ à 26,5) à 41,1 (IC à $99 \%$ : 35,6 à 47,5) (figure 6).

La même tendance à la hausse a été observée pour le taux de mortalité par cancer du foie et des voies biliaires intrahépatiques chez les hommes. Il est passé de 5,2 par 100000 (IC à $99 \%: 3,5$ à 5,2) en 1981 à 8,5 (IC à $99 \%: 7,4$ à 9,9) en 2009 (données provisoires) (figure 7).

Le taux d'hospitalisation de courte durée par les MCF a diminué de 1991 à 1997 et est resté relativement stable au cours des années suivantes (figure 8).

\section{Analyse}

Si l'on se fie à nos résultats, la tendance à la hausse de l'obésité dans la population québécoise est alarmante, surtout chez les jeunes hommes. Ces chiffres autorapportés, puisés dans les ESCC, pourraient être sous-estimés de 4 à $8 \%$ par 
FIGURE 2

Proportion de la population de 12 ans et plus ayant pris $\mathbf{1 4}$ consommations d'alcool et plus au cours d'une période de sept jours selon l'âge et le sexe, Québec, ESCC, 2000-2005

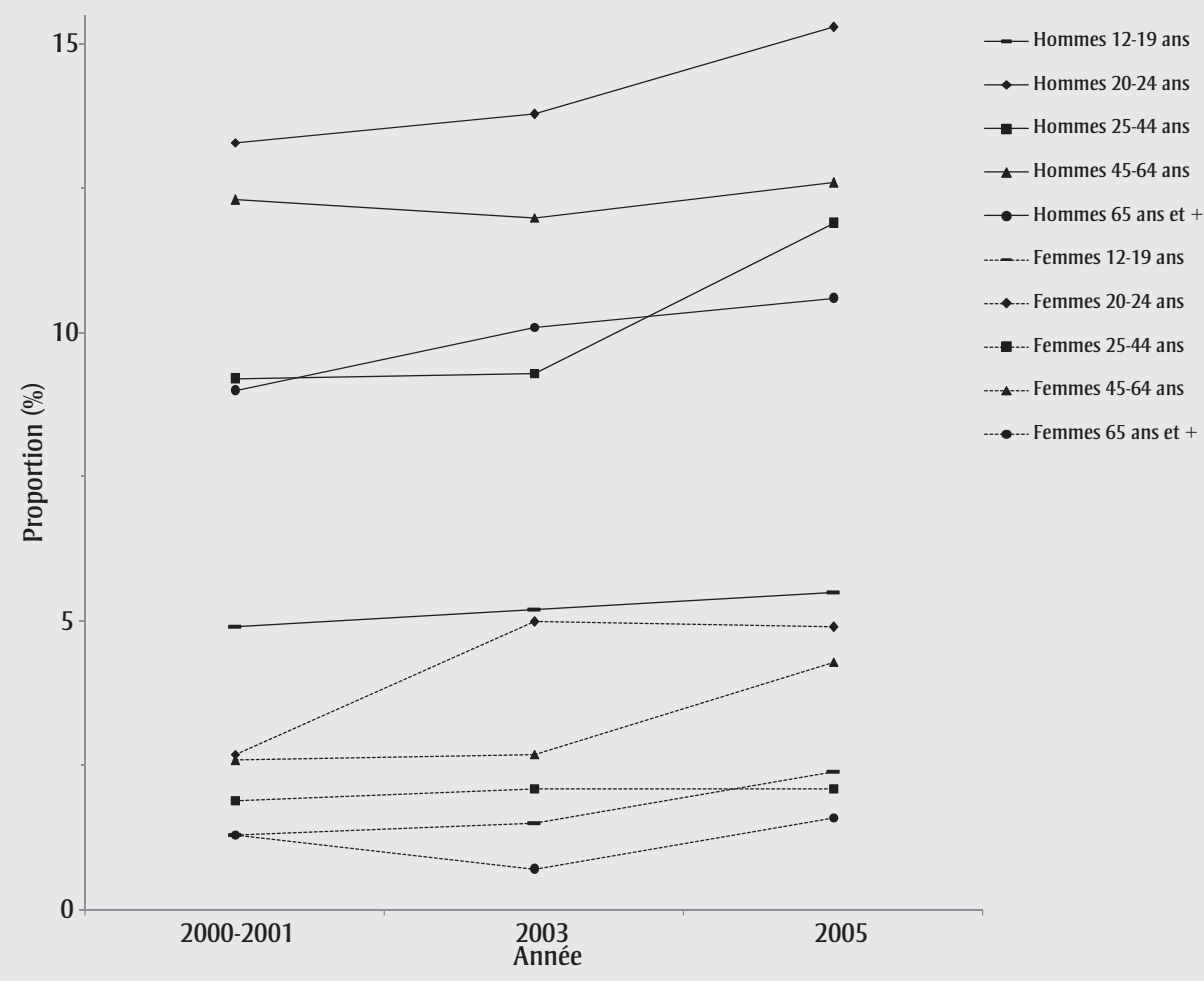

Abréviation : ESCC, Enquête sur la santé dans les collectivités canadiennes.

rapport aux études où l'on mesure directement les indicateurs d'obésitée ${ }^{24,25}$. Malgré cette limite, la tendance observée est en accord avec les résultats d'une étude publiée par l'ISQ ${ }^{25}$ basée sur des mesures directes dans laquelle on rapporte que la prévalence d'excès de poids (surplus de poids et obésité) chez les Québécois de 18 à 74 ans est passée de $43 \%$ en 1990 à $56 \%$ en $2004^{25}$. Le problème de l'obésité est d'une ampleur mondiale. Récemment, Finucane et collab. ${ }^{26}$ ont publié une étude où sont estimées les tendances de l'IMC dans 199 pays et territoires chez les adultes de 20 ans et plus. D’après ces auteurs, en 2008, la prévalence mondiale de l'obésité était de 9,8\% (IC à 95\%:9,0 à 10,0) chez les hommes et de $13,8 \%$ (IC à $95 \%: 13,1$ à 14,7) chez les femmes, soit près du double de la prévalence observée en 1980, qui était alors de 4,8 \% (IC à $95 \%: 4,0$ à 5,7) chez les hommes et de $7,9 \%$ (IC à $95 \%$ : 6,8 à 9,3) chez les femmes. L'association entre l'excès de poids et le risque d'acquérir une MCF a aussi été mesurée. Larsson et collab. ${ }^{27}$, grâce à une méta-analyse, ont rapporté que l'excès de poids est associé à une augmentation du risque de cancer du foie. Ces auteurs ont observé une hausse du risque de $17 \%$ chez les personnes présentant un embonpoint et de $89 \%$ chez celles présentant une obésité par rapport à celles ayant un poids normal. Chez les personnes souffrant d'obésité, le risque relatif (RR) était significativement plus élevé chez les hommes (RR: 2,42; IC à $95 \%: 1,83$ à 3,20) que chez les femmes (RR : 1,67; IC à $95 \%: 1,37$ à 2,03) ${ }^{27}$. Des études ultérieures seront nécessaires afin de mieux analyser l’obésité comme facteur de risque pour d'autres MCF comme le foie gras non alcoolique ${ }^{4,28,29}$.

L'alcool est une cause connue de cirrhose qui prédispose à un $\mathrm{CHC}^{30}$. Selon un rapport publié en 2010 concernant la consommation d'alcool et la santé publique des Québécois, les volumes de vente d'alcool enregistrés au Québec sont en hausse depuis la fin des années $1990^{31}$. Pendant la période de notre étude, la proportion totale des buveurs d'alcool est restée stable mais la répartition de buveurs selon la fréquence et le niveau (élevé) de consommation est à la hausse. Ces tendances sont plus remarquables chez les jeunes et chez les femmes. Des donnés similaires ont été rapportées par d'autres auteurs ${ }^{32}$.

Les infections chroniques avec le VHB et le VHC sont des causes connues de cirrhose et de CHC. Elles seraient responsables de 80 à $90 \%$ des cas de $\mathrm{CHC}$ dans le monde ${ }^{33}$. Le Canada est considéré comme un pays à basse endémicité pour ces deux infections. Selon l'ASPC, 0,7 à $0,9 \%$ de la population canadienne est infectée de façon chronique par le VHB. Le taux de déclaration du VHB a considérablement diminué entre la période de 1990 à 2007, passant de 11 par 100000 habitants (IC à $95 \%: 6,0$ à 18,1 ) à 3,3 par 100000 (IC à $95 \%$ : 1,6 à 4,8). Au Québec, on a observé la même évolution, avec une diminution marquée du taux d'incidence des cas aigus de VHB. Cela 
FIGURE 3

Nombre de cas d'hépatite virale B (VHB) selon la classe, nombre de cas d'hépatite virale C (VHC) et taux d'incidence annuelle de VHB aiguë, Québec, 1990-2009

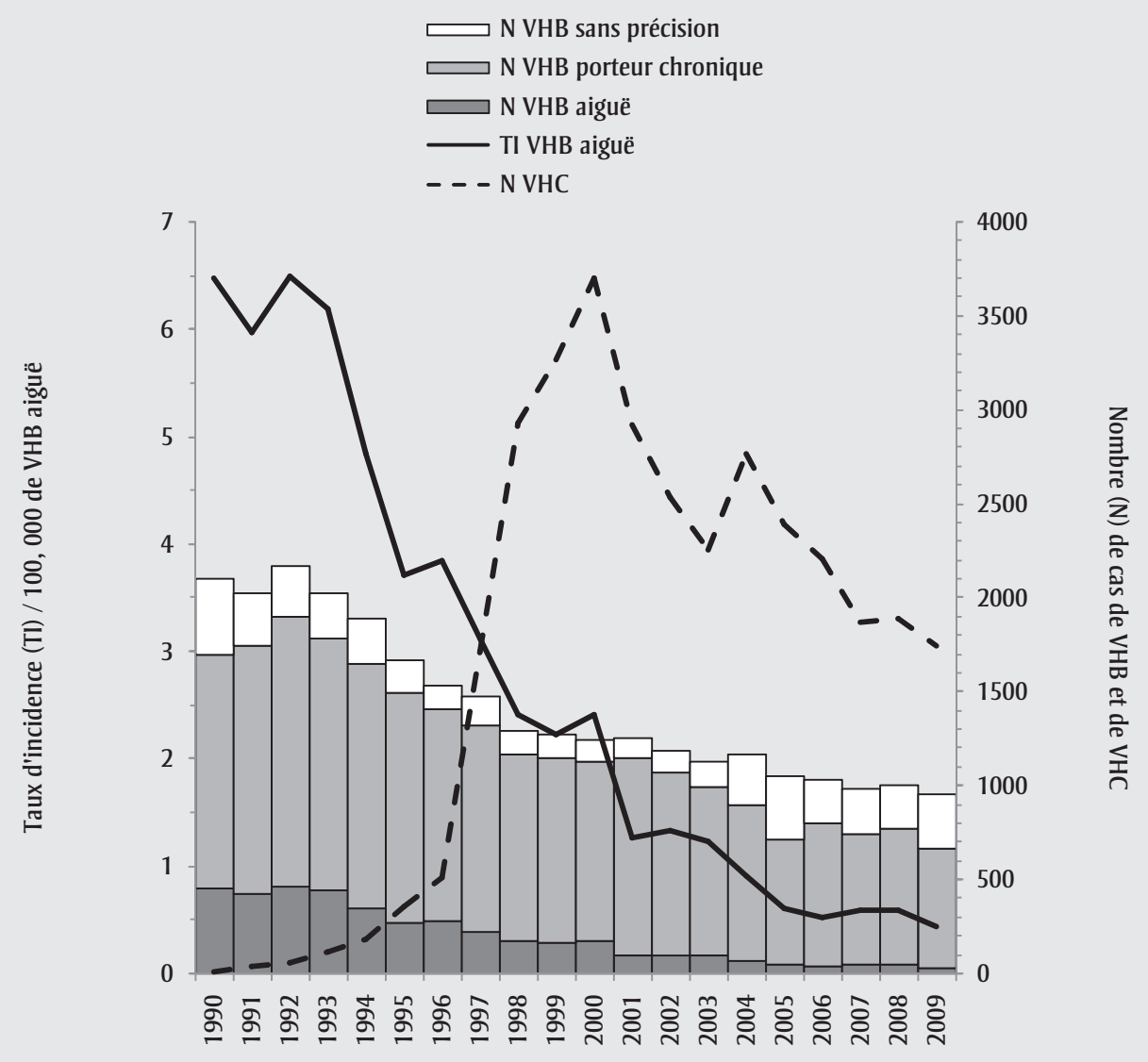

Année de réception de la déclaration

Abréviations : VHB, hépatite virale B; VHC, hépatite virale C.

peut être associé à un impact positif de l'immunisation élargie contre le VHB, dont le programme de vaccination scolaire introduit en $1994^{34}$.

Les chiffres sur les cas déclarés de VHC doivent être interprétés avec prudence. Une bonne partie de l'augmentation observée au cours des années 1990 est vraisemblablement due au meilleur accès aux tests de détection de cette infection et à un dépistage accru chez les populations à risque élevé d'infection. La diminution observée au cours des années 2000 pourrait être liée aux procédures de déduplication d'épisodes enregistrés dans le système MADO, intensifiées en 2004 et appliquées rétrospectivement et prospectivement, ainsi qu'à des activités de prévention chez les groupes à risque, notamment chez les utilisateurs de drogues injectables. Bien que la prévention de la transmission du VHB et du VHC fassent partie des axes d'intervention du Programme national de santé publique $2003-2012^{35}$, si l'on prend en compte qu'entre 75 et $85 \%$ des infections au VHC deviennent chroniques, 5 à $20 \%$ de celles-ci évolueront vers une cirrhose du foie et entre 1 et $5 \%$ mourront à cause de l'infection chronique (cirrhose ou $\mathrm{CHC})^{36}$, ce qui marque la pertinence des mesures intégrales de prise en charge pour ces patients.

Pour la période 1981-1999, le taux global de mortalité par maladies hépatiques chroniques et cirrhose a diminué de $42 \%$. Ces données sont similaires à celles de certains pays d'Europe dont l'Italie,
l'Espagne et le Portugal, qui rapportent des réductions de 25 à $30 \%$ depuis les années 1970. Elles contrastent avec celles de l'Angleterre et de l'Écosse qui présentent entre $35 \%$ et $112 \%$ d'augmentation du taux de mortalité par cirrhose du foie ${ }^{9}$. Les politiques moins restrictives sur l'alcool dans ces pays ont été identifiées comme faisant partie des causes importantes d'augmentation de cette mortalité. $\mathrm{Au}$ Canada, la diminution observée serait en lien avec une meilleure prise en charge des patients atteints ainsi que des politiques plus restrictives sur les ventes d'alcool et sur l'accès aux boissons alcoolisées. Après 2000, les taux de mortalité par cirrhose du foie et maladie alcoolique du foie fluctuent, en restant plus au moins dans la tendance à la baisse illustrée ailleurs. 
FIGURE 4

Taux de mortalité par maladies chroniques et cirrhose du foie selon le sexe, Québec, 1981-1999

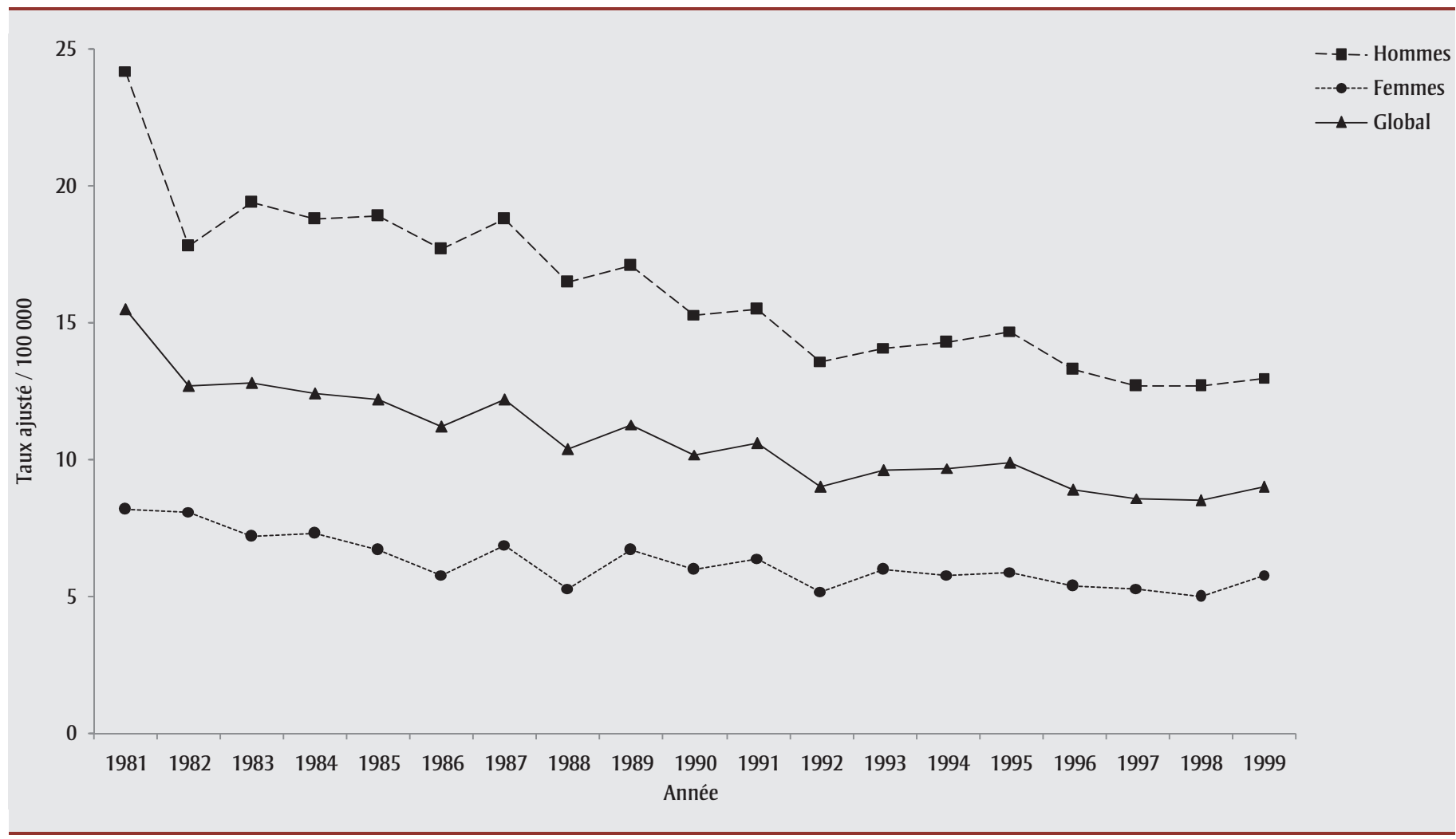

Au cours des dernières années, de nombreuses études ont signalé que l'incidence du CHC va en augmentation ${ }^{2,32,37-41}$, et le Québec n’est pas épargné par cette situa- tion. Pendant la période 1998-2007, le Québec a eu un taux d'incidence du cancer du foie de 3,7 par 100000 (IC à $95 \%: 3,5$

à 3,8$)$, au $2^{\mathrm{e}}$ rang au Canada après la
Colombie-Britannique $(3,9$; IC à $95 \%: 3,7$ à 4,1$)^{37}$ (données disponibles sur demande). D’auprès nos résultats, cette hausse débute après la période 1989-1991,

FIGURE 5

Taux de mortalité par maladie alcoolique du foie (MAF) et par fibrose et cirrhose hépatique (FCH) selon le sexe, Québec, 2000-2007

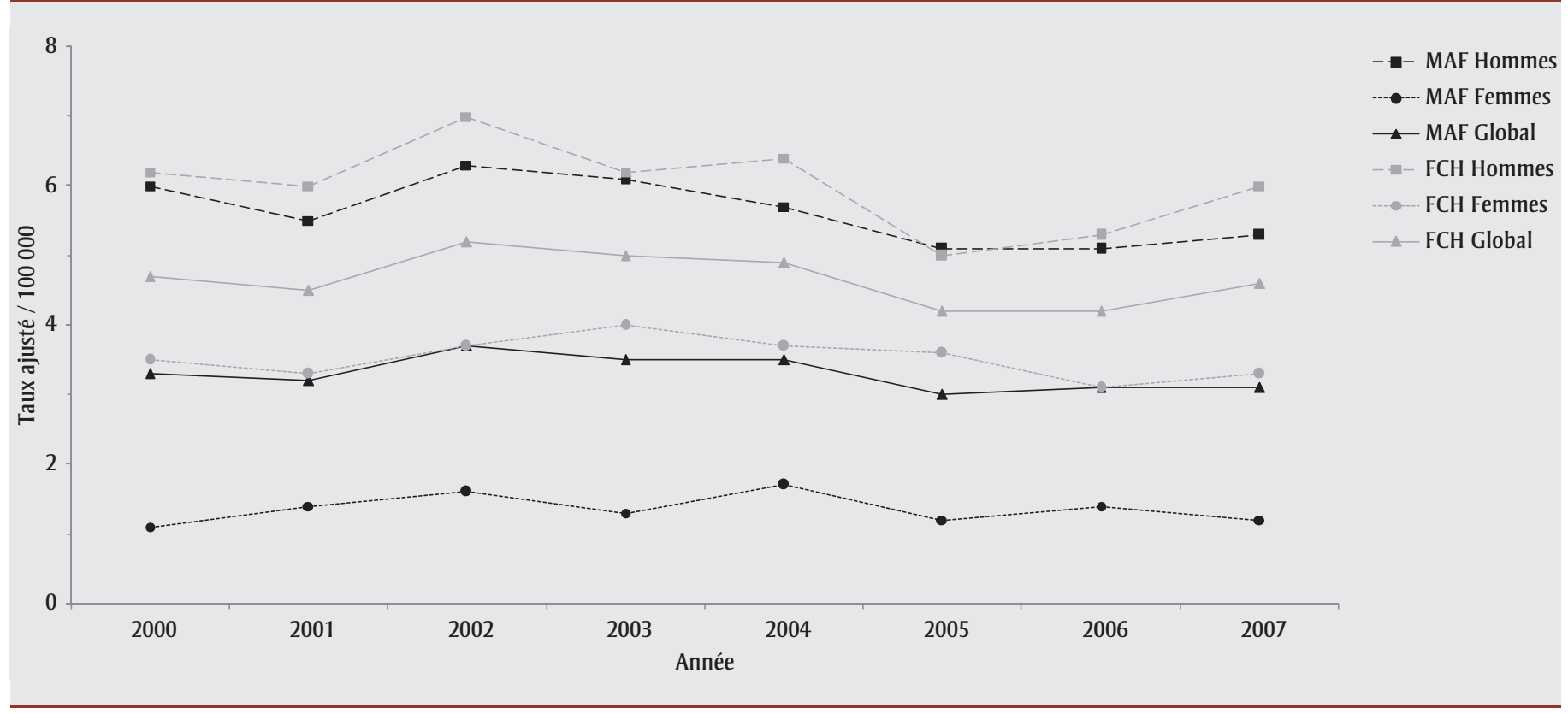

Abréviations : FCH, fibrose et cirrhose hépatique; MAF, maladie alcoolique du foie. 
FIGURE 6

Taux d'incidence du cancer du foie par période de trois ans selon l'âge, Québec, 1986-2006

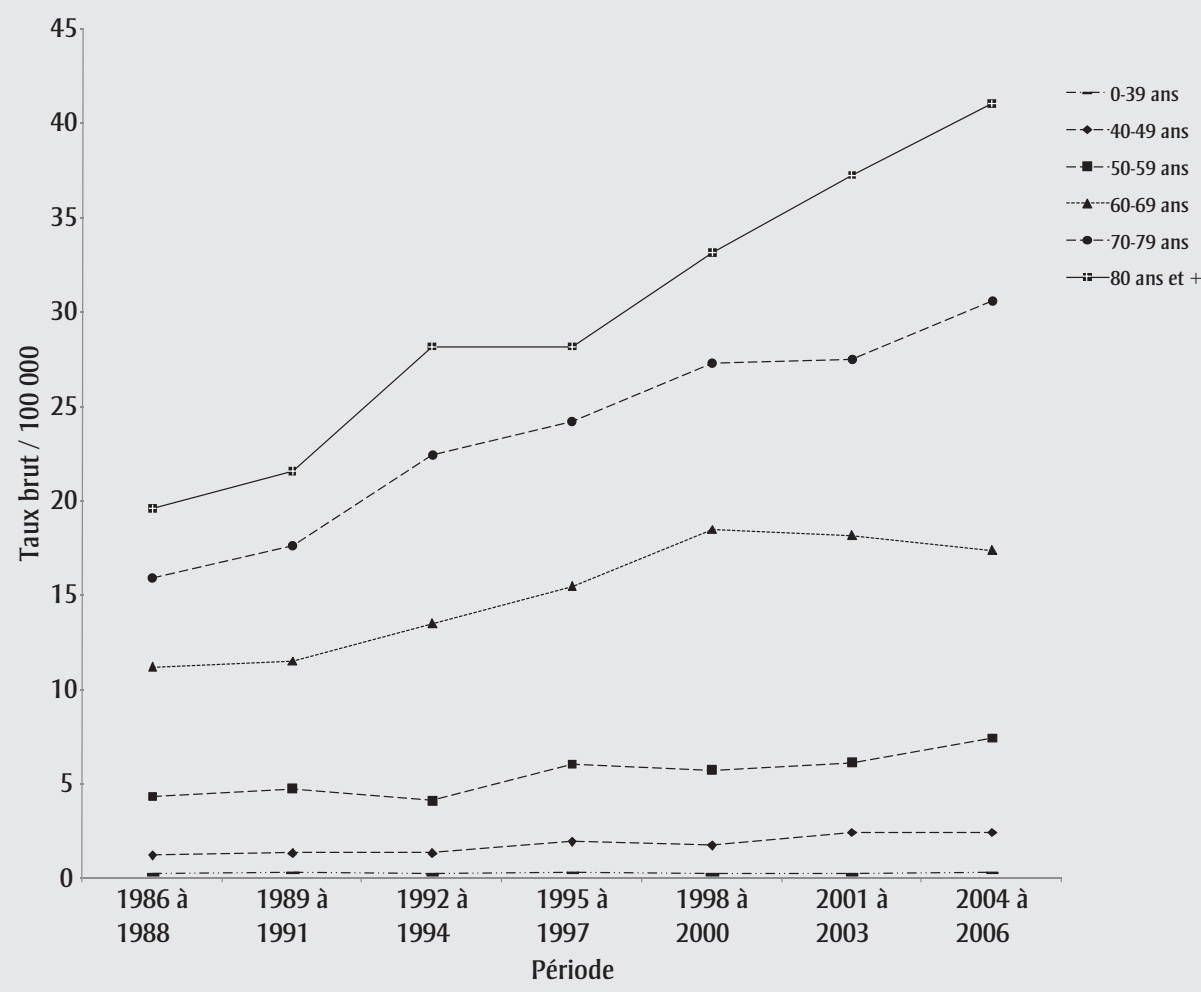

elle est constante chez les deux sexes et elle est particulièrement importante chez les sujets de 50 ans et plus. D'autre part, le taux de mortalité par cancer du foie suit celui de l'incidence, soulignant la haute létalité de ce type de tumeur ${ }^{2,32,37-41}$. Les tendances vers la hausse de l'incidence et de la mortalité du cancer du foie au
Québec accompagnent celles observées dans le reste du Canada. Selon la Société canadienne du cancer ${ }^{42}$, pendant la période 1997-2006, les taux d'incidence et de mortalité du cancer du foie chez les hommes ont subi un changement statistiquement significatif (respectivement de $3,1 \%$ et de $2,2 \%$ par année; ce change- ment est considéré comme statistiquement significatif à $2 \%$ ou plus par année $)^{42}$. Cette hausse concorde avec celles rapportées aux États-Unis, au Royaume-Uni et dans la plupart des pays européens ${ }^{9,39,43}$. L'augmentation du taux d'incidence du cancer du foie observée chez les plus âgés pourrait être en relation avec un effet de

FIGURE 7

Taux de mortalité par cancer du foie et des voies biliaires intrahépatiques selon le sexe, Québec, 1981-1999

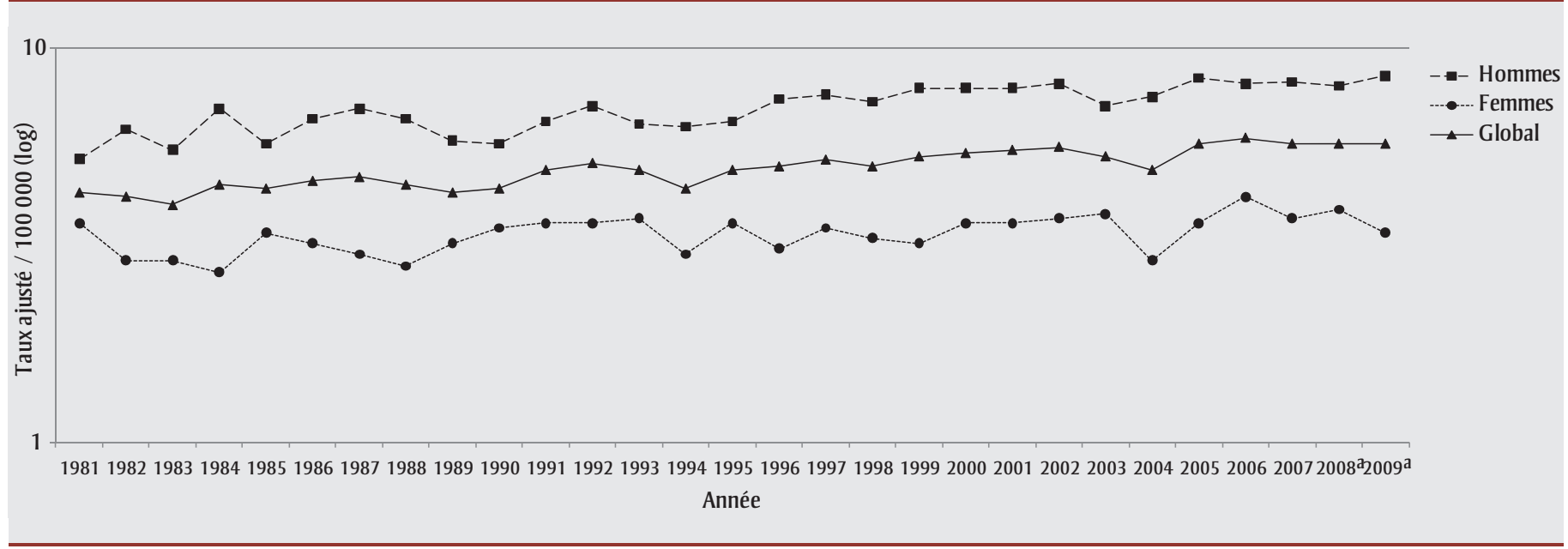

${ }^{\mathrm{a}}$ Données provisoires. 
FIGURE 8

Taux d'hospitalisation en soins physiques de courte durée pour maladies du foie, selon le sexe, Québec, 1990-2005

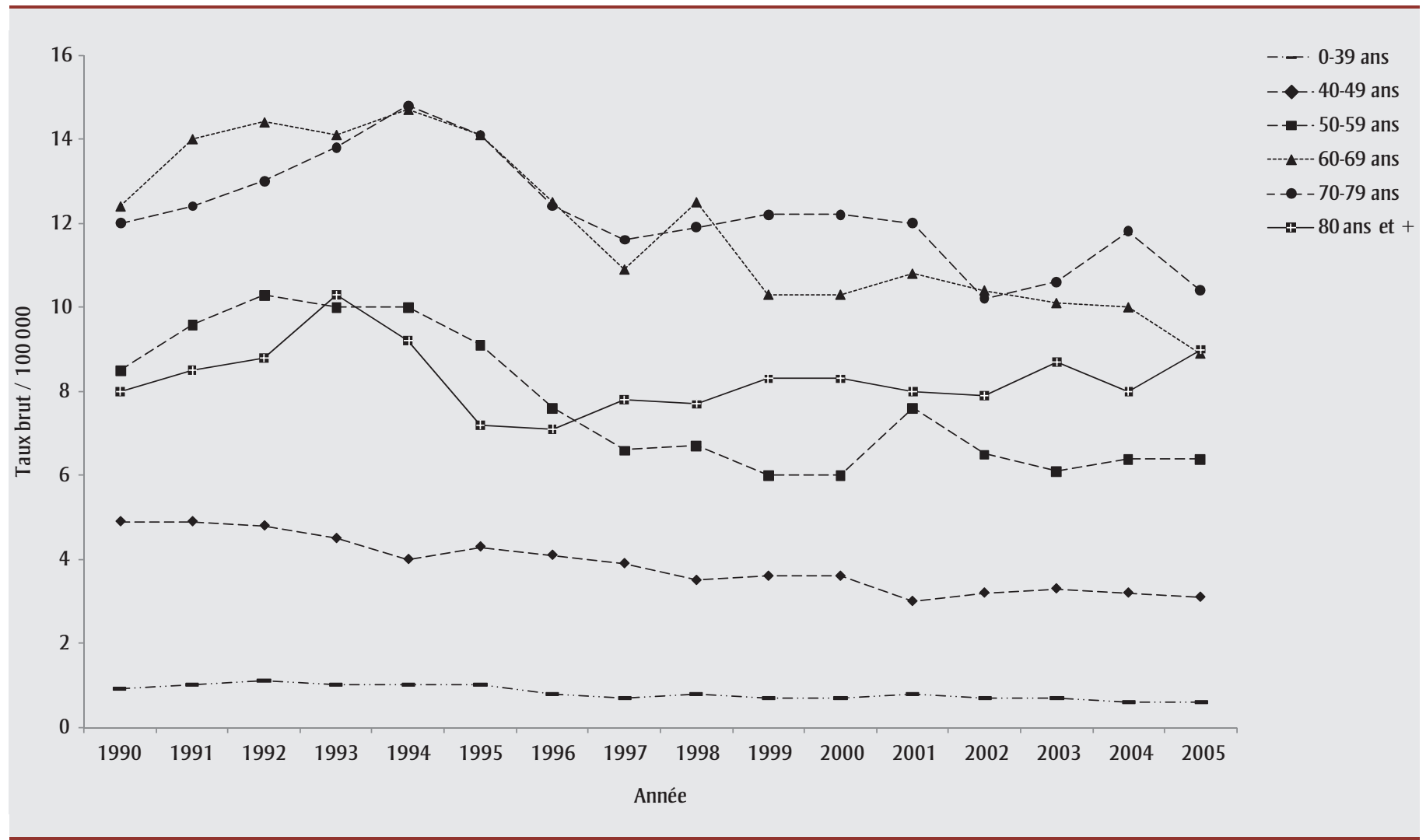

cohorte, et la meilleure prise en charge de ces patients pourrait probablement améliorer leur survie. La diminution observée du taux de mortalité due à la cirrhose du foie pourrait être causée par les progrès thérapeutiques (ex. : traitements antiviraux contre le VHB et le VHC plus accessibles et efficaces, greffes hépatiques) et pourraient éventuellement se répercuter sur les tendances concernant le CHC.

La diminution du taux d'hospitalisation en soins physiques de courte durée pour les maladies du foie au Québec à la fin des années 1990 pourrait être liée au virage ambulatoire des services de soins, instauré comme mesure de maîtrise des dépenses en santé, entre autres raisons ${ }^{44}$.

Malgré les concordances avec les résultats d'autres auteurs, notre étude comporte des limites à considérer lors de l'interprétation des résultats. Il n'est pas possible, sur la base de nos résultats, de départager d'attribution des divers facteurs de risque des différentes MCF. Le niveau d'accès octroyé pour consulter les sources de données n'a pas permis une ventilation plus détaillée des épisodes ou des affections d'intérêt. Les données des ESCC ont des limites non négligeables dans la comparabilité intra- et inter-cycles dues aux changements dans la méthodologie, et ce, malgré l'existence de lignes directrices pour la comparabilité et l'interprétation des indicateurs ${ }^{35,45,46}$. Notre étude observationnelle ayant une portée descriptive des tendances, en raison du temps et des ressources disponibles, plusieurs aspects relatifs aux mesures d'association et à la recherche de différences significatives entre les périodes et les populations étudiées n'ont pas été explorés. Nous avons privilégié une vision globale des problèmes des MCF étudiés, en faisant des liens entre les déterminants et les états morbides. Tout en demeurant simple, nous pensons que cette approche est pertinente dans le cadre de ce type de problématique : elle permet de formuler des hypothèses qui pourront être validées dans des études futures et de souligner les aspects les plus importants pour la prise de décision.

\section{Remerciements}

Nous remercions l'Institut national de santé publique du Québec et l'Hôpital universitaire de Bellvitge pour leur soutien dans la réalisation du stage qui a permis de mener à terme cette étude.

Il n’y a ni conflit d'intérêt, ni source de financement en lien avec le matériel présenté dans cet article.

\section{Références}

1. Bell BP, Manos MM, Zaman A, Terrault N, Thomas A, Navarro VJ et collab. The epidemiology of newly diagnosed chronic liver disease in gastroenterology practices in the United States: results from populationbased surveillance. Am J Gastroenterol. 2008;103(11):2727-36.

2. Sanyal AJ, Yoon SK, Lencioni R. The etiology of hepatocellular carcinoma and consequences for treatment. Oncologist. 2010;15 Suppl 4:14-22. 
3. Severi T, van MH, Verslype C, van Pelt JF. Tumor initiation and progression in hepatocellular carcinoma: risk factors, classification, and therapeutic targets. Acta Pharmacol Sin. 2010;31(11):1409-1420.

4. Marchesini G, Moscatiello S, Di DS, Forlani G. Obesity-associated liver disease. J Clin Endocrinol Metab. 2008;93(11 Suppl 1): S74-S80.

5. Corrao G, Bagnardi V, Zambon A, La VC. A meta-analysis of alcohol consumption and the risk of 15 diseases. Prev Med. 2004;38(5):613-619.

6. Tanaka K, Tsuji I, Tamakoshi A, Matsuo K, Ito $\mathrm{H}$, Wakai $\mathrm{K}$ et collab. Obesity and Liver Cancer Risk: An Evaluation Based on a Systematic Review of Epidemiologic Evidence Among the Japanese Population. Jpn J Clin Oncol. 2012;42(3):212-221.

7. Fattovich G, Stroffolini T, Zagni I, Donato F. Hepatocellular carcinoma in cirrhosis: incidence and risk factors. Gastroenterology. 2004;127(5 Suppl 1):S35-S50.

8. Berzigotti A, Garcia-Tsao G, Bosch J, Grace ND, Burroughs AK, Morillas R et collab. Obesity is an independent risk factor for clinical decompensation in patients with cirrhosis. Hepatology. 2011;54(2):555-561.

9. Leon DA, McCambridge J. Liver cirrhosis mortality rates in Britain, 1950 to 2002. Lancet. 2006;367(9511):645.

10. Murphy SL. Deaths: final data for 1998. Natl Vital Stat Rep. 2000 Jul 24;48(11):1-105.

11. Anderson RN, Smith BL. Deaths: leading causes for 2002. Natl Vital Stat Rep. 2005;53(17):1-89.

12. Kung HC, Hoyert DL, Xu J, Murphy SL. Deaths: final data for 2005. Natl Vital Stat Rep. 2008;56(10):1-120.

13. Murphy SL, Xu JQ, Kochanek KD. Deaths: Preliminary Data for 2010. Natl Vital Stat Rep. 2012;60(4).

14. Statistique Canada. 2008. Mortalité : liste sommaire des causes. Périodicité annuelle. 2011. ( $N^{\circ} 84 \mathrm{~F} 0209 \mathrm{X}$ au catalogue)

15. Lim SG, Mohammed R, Yuen MF, Kao JH. Prevention of hepatocellular carcinoma in hepatitis B virus infection. J Gastroenterol Hepatol. 2009;24(8):1352-1357.
16. Asia-Pacific Working Party on Prevention of Hepatocellular Carcinoma. Prevention of hepatocellular carcinoma in the AsiaPacific region: consensus statements. J Gastroenterol Hepatol. 2010;25(4):657-663.

17. Gouvernement du Québec. Infocentre [Internet]. 2010. Consultable à partir de la page : www.infocentre.inspq.rtss.qc.ca

18. Statistique Canada. Enquête sur la santé dans les collectivités canadiennes (ESCC), cycle 2.1 (2003). Guide du ficher de microdonnées à grande diffusion [Internet]. Ottawa: Statistique Canada. 2005. PDF (700 Ko) téléchargeable à partir du lien : http://www.portail-rrsstq.com/export/sites /rrsstq/fiches/docs/ESCC_guide_C2.1.pdf

19. Ministère de la Santé et des Services sociaux. Surveillances des maladies à déclaration obligatoire au Québec. Définitions nosologiques. Maladies d'origine infectieuse. $8^{\mathrm{e}}$ éd [Internet]. Québec : MSSS. 2011. PDF (15,4 Mo) téléchargeable à partir du lien : http://publications.msss .gouv.qc.ca/acrobat/f/documentation/2011 /11-268-01.pdf

20. Agence de la santé publique du Canada. Définitions nosologiques des maladies transmissibles faisant l'objet d'une surveillance nationale. Relevé des maladies transmissibles au Canada. 2009;35(s2):1-139.

21. Ministère de la Santé et des Services sociaux. Service du développement de l'information. La population du Québec par territoire des centres locaux de services communautaires, par territoire des réseaux locaux de services et par région sociosanitaire, de 1981 à 2031 [Internet]. Québec : MSSS. 2010. Consultable aux pages : http://msss.gouv.qc.ca/statistiques /stats_sss/index.php?population - Estimations: http://msss.gouv.qc.ca/statistiques/stats_sss /index.php?id = 120,0,0,1,0,0 - Projections: http://msss.gouv.qc.ca/statistiques/stats_sss /index.php?id = 121,0,0,1,0,0.

22. Christiansen T, Bruus M, Lauritsen JM, Kreiner S. EpiData Analysis. V2.2.1.171. Data management and statistical analysis package [Internet]. EpiData Association. 2009. Consultable à partir de la page : http://www.epidata.dk
23. Statistique Canada. Enquête sur la santé dans les collectivités canadiennes (ESCC) cycle 3.1 (2005). Ficher de microdonnées à grande diffusion (FMGD). Guide de l'utilisateur [Internet]. Ottawa: Statistique Canada. 2006. [Document archivé. Disponible sur demande à Statistique Canada]

24. Institut national de santé publique du Québec et ministère de la Santé et des Services sociaux du Québec, en collaboration avec l'Institut de la statistique du Québec. Portrait de santé du Québec et de ses régions 2006 : les analyses - Deuxième rapport national sur l'état de santé de la population du Québec [Internet]. Québec : INSPQ. 2006. PDF (2,2 Mo) téléchargeable à partir du lien : http://www.inspq.qc.ca/pdf/publications /546-PortraitSante2006_Analyses.pdf

25. Audet N. L'évolution de l'excès de poids chez les adultes québécois de 1990 à 2004: mesures directes. Zoom Santé [Internet]. Québec : Institute de la statistique du Québec. 2007. PDF (353 Ko) téléchargeable à partir du lien : www.stat.gouv.qc.ca /publications/sante/pdf2007/zoom_sante _juin07.pdf

26. Finucane MM, Stevens GA, Cowan MJ, Danaei G, Lin JK, Paciorek CJ, et al. National, regional, and global trends in body-mass index since 1980: systematic analysis of health examination surveys and epidemiological studies with 960 countryyears and 9.1 million participants. Lancet. 2011;377(9765):557-567.

27. Larsson SC, Wolk A. Overweight, obesity and risk of liver cancer: a meta-analysis of cohort studies. Br J Cancer. 2007;97(7):1005-1008.

28. Bellentani S, Scaglioni F, Marino M, Bedogni G. Epidemiology of non-alcoholic fatty liver disease. Dig Dis. 2010;28(1):155-161.

29. Scheen AJ, Luyckx FH. Obesity and liver disease. Best Pract Res Clin Endocrinol Metab. 2002;16(4):703-716.

30. World Cancer Research Fund / American Institute for Cancer Research. Food, Nutrition, Physical Activity, and the Prevention of Cancer: a Global Perspective [Internet]. Washington DC: AICR. 2007. PDF $(13,4 \mathrm{Mo})$ téléchargeable à partir du lien : http://www.dietandcancerreport.org /cancer_resource_center/downloads/Second _Expert_Report_full.pdf 
31. April N, Begin C, Morin R. La consommation d'alcool et la santé publique au Québec [Internet]. Québec : INSPQ. 2010. Consultable à partir de la page : http://www.inspq.qc.ca

32. Nordenstedt H, White DL, El-Serag HB. The changing pattern of epidemiology in hepatocellular carcinoma. Dig Liver Dis. 2010;42 Suppl 3:S206-S214.

33. Agence de la santé publique du Canada. Hepatitis B infection in Canada. Ottawa : Agence de la santé publique du Canada ; 2008.

34. Gilca V, Duval B, Boulianne N, Dion R, De Serres G. Impact of the Quebec schoolbased hepatitis B immunization program and potential benefit of the addition of an infant immunization program. Pediatr Infect Dis J. 2006;25(4):372-374.

35. Ministère de la Santé et des Services sociaux. Programme national de santé publique 2003-2012 [Internet]. Québec : INSPQ. 2003. PDF (1,2 Mo) téléchargeable à partir du lien : http://publications.msss .gouv.qc.ca/acrobat/f/documentation/2002 /02-216-01.pdf

36. World Health Organization. Global alert and reponse. Hepatitis C [Internet]. 2002. PDF (259 Ko) téléchargeable à partir du lien : http://www.who.int/csr/disease/hepatitis /Hepc.pdf

37. Agence de la santé publique du Canada. Site de Surveillance de cancer en direct. Ottawa : ASPC.

38. Ferlay J, Shin HR, Bray F, Forman D, Mathers C, Parkin DM. Estimates of worldwide burden of cancer in 2008: GLOBOCAN 2008. Int J Cancer. 2010;127(12):2893-2917.

39. Jemal A, Siegel R, Xu J, Ward E. Cancer statistics, 2010. CA Cancer J Clin. 2010; 60(5):277-300.

40. Marcos-Gragera R, Cardo X, Galceran J, Ribes J, Izquierdo A, Borras J. [Cancer incidence in Catalonia, 1998-2002]. Med Clin (Barc ). 2008;131 Suppl 1:4-10.

41. Michielsen PP, Francque SM, van Dongen JL. Viral hepatitis and hepatocellular carcinoma. World J Surg Oncol. 2005;3:27.
42. Société canadienne du cancer. Comité directeur de la Société canadienne du cancer: Statistiques canadiennes sur le cancer 2010. Toronto : Société canadienne du cancer. 2010

43. Ribes J, Pareja L, Esteban L, Cleries R. [Analysis of cancer incidence, survival and mortality according to the main tumoral localizations, 1985-2019: liver cancer]. Med Clin (Barc). 2008;131 Suppl 1:83-87.

44. L'Association des praticiens de service social en milieu de santé du Québec. Le virage ambulatoire et le service social en milieu de santé. Réflexions et propositions [Internet]. Service social. 1996:45(3);17591. PDF téléchargeable à partir du lien : http://www.erudit.org/revue/ss/1996/v45 /n3/706744ar.pdf

45. Institut national de santé publique du Québec et ministère de la Santé et des Services sociaux du Québec en collaboration avec l'Institut de la statistique du Québec. Guide spécifique des aspects méthodologiques des données d'enquêtes sociosanitaires du Plan commun de surveillance - Enquête sur la santé dans les collectivités canadiennes, cycles 1.1, 2.1, 3.1 et 2007-2008 [Internet]. Québec : INSPQ. 2011. PDF (1,8 Mo) http: //www.stat.gouv.qc.ca/publications/sante /doc_technique/ESCC_guide_methodologique _mai2011.pdf

46. Plante N, Côté L, Courtemanche R, Nanhou $\mathrm{V}$. Incidence des changements méthodologiques de l'Enquête sur la santé dans les collectivités canadiennes sur la comparabilité entre les cycles 1.12 .1 et 3.1 aux niveaux provincial et régional de même que sur la comparabilité régionale intra cycle Québec Institut de la statistique du Québec [Internet]. Québec : Institut de la statistique du Québec. 2010. PDF (393 Ko) téléchargeable à partir du lien : http: //www.stat.gouv.qc.ca/publications/sante /doc_technique/ESCC_comparabilite_cycles _11_21_21.pdf 